\title{
Effect of Admission Hyperglycemia on Short-Term Prognosis of Patients with Non-ST Elevation Acute Coronary Syndrome without Diabetes Mellitus
}

\author{
Wei Liu, ${ }^{1}$ Zhijuan Li, ${ }^{1}$ Shiying Xing, ${ }^{1}$ and Yanwei Xu $\mathbb{D}^{2}$ \\ ${ }^{1}$ Department of Cardiology, The First Affiliated Hospital of Henan University of Science and Technology, Luoyang 471003, China \\ ${ }^{2}$ School of Mechatronics Engineering, Henan University of Science and Technology, Luoyang 471003, China \\ Correspondence should be addressed to Yanwei Xu; xuyanweiluoyang@163.com
}

Received 23 August 2021; Revised 16 November 2021; Accepted 17 November 2021; Published 6 December 2021

Academic Editor: Gaetano Santulli

Copyright (๑) 2021 Wei Liu et al. This is an open access article distributed under the Creative Commons Attribution License, which permits unrestricted use, distribution, and reproduction in any medium, provided the original work is properly cited.

\begin{abstract}
Objective. To evaluate the effect of admission hyperglycemia on the short-term prognosis of patients with non-ST elevation acute coronary syndrome (NSTE-ACS) without diabetes mellitus. Methods. The clinical data of 498 patients with NSTE-ACS admitted to the Department of Cardiology of the First Affiliated Hospital of Henan University of Science and Technology between March 2018 and November 2020 were analyzed. Based on the blood glucose (BG) level at admission, patients were divided into three groups: A $(\mathrm{BG}<7.8 \mathrm{mmol} / \mathrm{L}), \mathrm{B}(7.8 \mathrm{mmol} / \mathrm{L} \leq \mathrm{BG}<11.1 \mathrm{mmol} / \mathrm{L})$, and $\mathrm{C}(\mathrm{BG} \geq 11.1 \mathrm{mmol} / \mathrm{L})$. The clinical data of the three groups were compared. Results. There was no significant difference between the three groups in terms of age, sex, hypertension, hyperlipidemia, smoking, and history of myocardial infarction $(p>0.05)$. However, there were significant differences in the incidences of multivessel disease, renal insufficiency, pump failure, and emergency percutaneous coronary intervention, and levels of high-sensitivity C-reactive protein, cardiac troponin $\mathrm{T}$, and creatine kinase isoenzyme MB among the three groups $(p<0.05$ for all). The incidences of severe pump failure, malignant arrhythmias, and death were significantly higher in groups B and C compared to group A $(p<0.05)$. Additionally, the incidences of severe pump failure, malignant arrhythmias, and death were significantly higher in group C compared to group B $(p<0.05)$. Multivariate logistic regression analysis showed that hyperglycemia, renal insufficiency, Killip grade III/IV, and age were risk factors of in-hospital death. Conclusion. Hyperglycemia at admission is a risk factor for adverse in-hospital clinical outcomes in patients with NSTE-ACS.
\end{abstract}

\section{Introduction}

Diabetes is an important independent risk factor for coronary atherosclerosis. Many previous studies have confirmed that hyperglycemia at admission is common in patients with acute coronary syndrome (ACS), and it is a risk factor for inhospital death and complications [1-4]. Previous epidemiological studies showed that $25-50 \%$ of ACS patients had elevated blood glucose (BG) level at admission. Recent studies suggest that the effects of hyperglycemia on the prognosis of ACS differ between diagnosed and undiagnosed diabetes. Hyperglycemia is a stronger predictor of adverse events in ACS patients without known diabetes than those with his- tory of diabetes $[5,6]$. Although there is a clear correlation between hyperglycemia and the occurrence and development of cardiovascular disease, the significance and treatment of hyperglycemia in ACS patients are not clear. The aim of this study was to investigate the effect of admission hyperglycemia on the short-term prognosis in patients with the non-ST elevation ACS (NSTE-ACS) without diabetes.

\section{Data and Methods}

2.1. Research Populations. ACS is a common, serious heart disease that comprises a group of syndromes due to rupture or invasion of the coronary atherosclerotic plaque and 
secondary complete or incomplete occlusive thrombosis. ACS includes acute ST-segment elevation myocardial infarction, acute non-ST-segment elevation myocardial infarction, and unstable angina (UA). The clinical classification of the acute coronary syndrome is shown in Figure 1.

This was a retrospective analysis of prospectively enrolled consecutive NSTE-ACS patients treated at the Department of Cardiology of the First Affiliated Hospital of Henan University of Science and Technology from March 2018 to November 2020. NSTE-ACS was diagnosed on the basis of typical angina symptoms lasting for $>10$ minutes, accompanied by at least one of the following: ST-segment depression $\geq 0.5 \mathrm{~mm}, 0.5-1.0 \mathrm{~mm}$ transient ST-segment elevation in two consecutive leads for $<30$ minutes, $\mathrm{T}$-wave inversion $>1 \mathrm{~mm}$ before the chest pain or within 12 hours after the chest pain, and/or myocardial enzymes (cardiac troponin $\mathrm{T}(\mathrm{TnT})$ or creatine kinase isozymes $\mathrm{MB}$ (CK$\mathrm{MB})$ ) exceeding the upper limit of the normal values. Patients were excluded if they had past diabetes, incomplete clinical and coronary angiography data, admission BG level $>20 \mathrm{mmol} / \mathrm{L}$, symptoms of ketoacidosis, and/or new-onset diabetes. A total of 498 patients with NSTE-ACS and high BG without history of diabetes were enrolled. The Ethical Committee of the First Affiliated Hospital of Henan University of Science and Technology approved the study protocol.

2.2. Definition. Hypertension is a syndrome characterized by increased systemic arterial blood pressure (systolic pressure $\geq 140 \mathrm{mmHg}$ and diastolic pressure $\geq 90 \mathrm{mmHg}$ ), which can be accompanied by functional or organ damage to the heart, brain, kidney, and/or other organs. Hypertension is the most common chronic disease and the main risk factor of cardiovascular and cerebrovascular diseases. Hypertension is divided into three grades according to severity: mild (systolic blood pressure: $140-159 \mathrm{mmHg}$ or diastolic blood pressure: $90-99 \mathrm{mmHg}$ ), moderate (systolic blood pressure: $160-179 \mathrm{mmHg}$ or diastolic blood pressure: $100-109 \mathrm{mmHg}$ ), and severe (systolic blood pressure: $\geq 180 \mathrm{mmHg}$ or diastolic blood pressure: $\geq 110 \mathrm{mmHg}$ ).

Hyperlipidemia refers to high blood lipid level, which can cause serious diseases, such as atherosclerosis, coronary heart disease, and pancreatitis. Hyperlipidemia usually is divided into hypertriglyceridemia, hypercholesterolemia, mixed hyperlipidemia, and low high-density lipoprotein cholesterol. Hypercholesterolemia is related to age and gender of patients. The majority of triglycerides are associated with genetic and environmental changes, and metabolic disorders. Most cases of hypertriglyceridemia are usually associated with abnormal BG, and diabetes mellitus is associated with hypertriglyceridemia.

Heart failure refers to systolic and/or diastolic function of the heart, leading to impaired pumping of the blood returned from the venous circulation to the heart, causing blood stasis in the venous system, insufficient perfusion in the arterial system, and cardiac circulation disorder syndrome. This disorder manifests as pulmonary and vena cava congestion. Heart failure is not an independent disease, but the final stage of several heart diseases. The vast majority of cases of heart failure affect the left side of the heart, and the first manifestation is often pulmonary congestion. Severe pump failure is defined as New York Heart Association (NYHA) classification grade $\geq 3$. Target lesion revascularization (TLR) is defined as repeated interventional therapy or bypass surgery within the stent and within $5 \mathrm{~mm}$ of the proximal and distal ends of the stent. Malignant arrhythmia includes ventricular tachycardia, ventricular fibrillation, and high atrioventricular block.

2.3. Research and Treatment Methods. Patients with suspected ACS underwent an electrocardiogram (ECG) and measurement of the BG level (hexokinase method, Olympus AU400) and myocardial injury markers. ACS was classified by the cardiovascular doctor into unstable angina pectoris, acute ST-segment elevation myocardial infarction, and acute NSTE myocardial infarction based on the ECG and levels of myocardial injury markers. Emergency coronary angiography was performed on patients in a critical condition, such as intractable or recurrent angina pectoris with dynamic ST-segment changes, heart failure, life-threatening arrhythmia, or hemodynamic instability. Stents were implanted in these patients according to the disease condition after the target vessel was determined. Other patients in a less critical state underwent percutaneous coronary intervention (PCI). The research scheme of this paper is shown in Figure 2.

After admission to the Department of Cardiology of the First Affiliated Hospital of Henan University of Science and Technology, the BG level of the patients was checked and ACS was classified into different types. We identified 754 NSTE-ACS with hyperglycemia but excluded 256 patients due to history of diabetes $(n=205)$, incomplete data $(n=24)$, BG level $>20 \mathrm{mmol} / \mathrm{L}(n=12)$, symptoms of ketoacidosis $(n=8)$, or new-onset diabetes mellitus $(n=7)$. Finally, 498 NSTE-ACS patients were included in the study.

Based on the BG level, NSTE-ACS patients were divided into three groups: A $(\mathrm{BG}<7.8 \mathrm{mmol} / \mathrm{L}), \mathrm{B}(7.8 \mathrm{mmol} / \mathrm{L} \leq$ $\mathrm{BG}<11.1 \mathrm{mmol} / \mathrm{L})$, and $\mathrm{C}(\mathrm{BG} \geq 11.1 \mathrm{mmol} / \mathrm{L})$. The risk factors for ACS were recorded for each group, including age, hypertension, hyperlipidemia, smoking history, clinical biochemical indexes, inflammatory markers, and left ventricular ejection fraction as measured by echocardiography. SPSS software (IBM Corp., Armonk, NY, USA) was used to perform statistical analysis. Differences were considered statistically significant at $p<0.05$.

2.4. Data Quality Control. The quality of the statistical data affects the research accuracy. Data quality control requires scientific and rigorous work. In the context of big data, the quality and efficiency of hospital data should be continuously improved.

All of the departments providing the data were incorporated into the information construction by our hospital to establish an ideal data quality management system. The hospital employees continuously regularly their skills and were familiar with the operation process of the information system. Our hospital regularly monitored the data operation and randomly checked the quality and standardization of the statistical to identify and solve potential problems. This ensured smooth information interaction in the hospital 


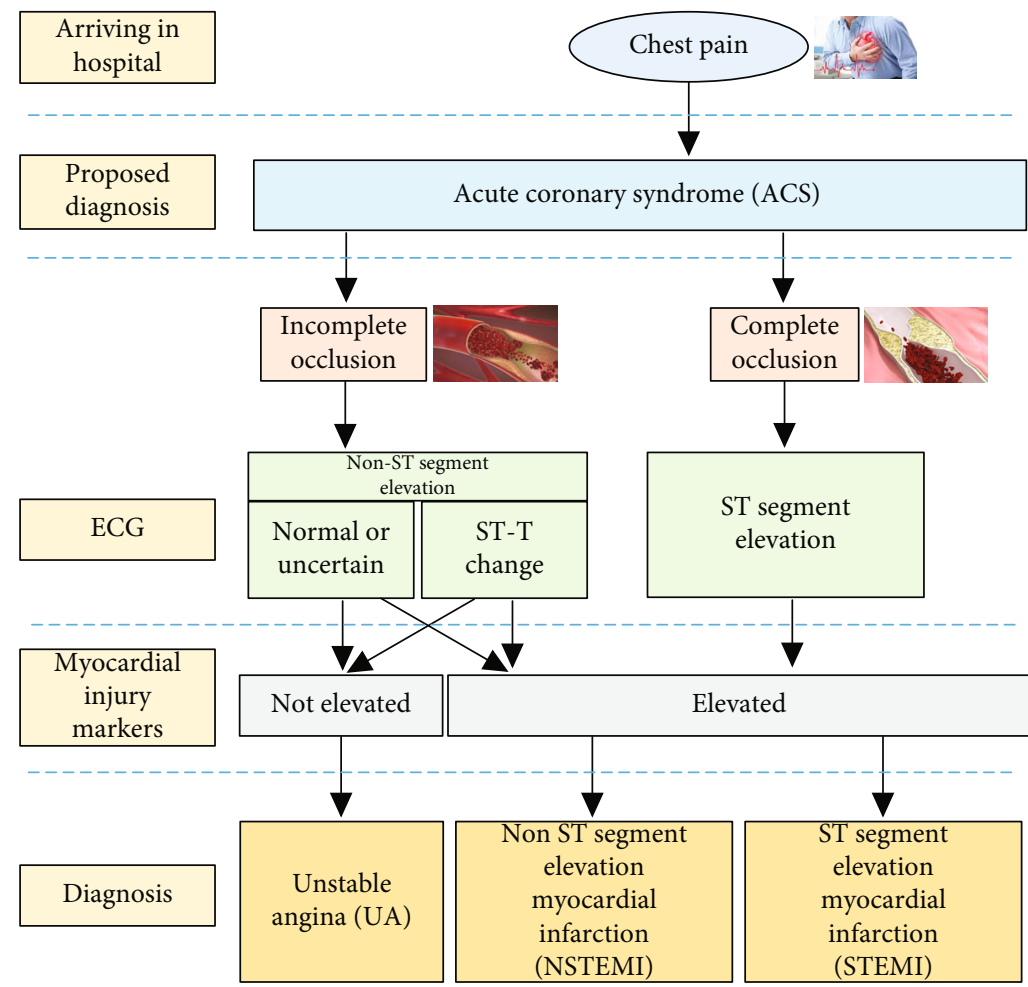

FIGURE 1: Classification of the acute coronary syndrome.

and improved the accuracy of the information. It also provided a solid foundation for the data quality control in our research.

2.5. Statistical Methods. SPSS software (version 25; IBM Corp., Armonk, NY, USA) was used to perform statistical analysis. Continuous variables are represented by $(\bar{x} \pm s)$, and the standard deviation $s$ is expressed as

$$
s=\sqrt{\frac{1}{N} \sum_{i=1}^{N}\left(x_{i}-\bar{x}\right)^{2}} .
$$

In (1), $s$ represents the standard deviation, $x_{i}$ represents the variable, and $\bar{x}$ represents the average value.

Categorical variables are expressed as numbers or percentages. Means between the multiple groups were compared using one-way ANOVA. The chi-square test was used to measure the difference between the theoretical and actual values, using the following formula:

$$
\chi^{2}=\sum \frac{\left(f_{o}-f_{e}\right)^{2}}{f_{e}} .
$$

In (2), $\chi^{2}$ represents the chi-square value, $f_{o}$ represents the actual value, and $f_{\mathrm{e}}$ represents the theoretical value.

Multivariate logistic stepwise regression was used to calculate the odds ratio (OR) for the predictors of in-hospital death and their impact on the outcomes.

\section{Results}

3.1. Comparison of Baseline Characteristics of the Three Groups. There was no significant difference among the three groups in terms of age, sex, hypertension, hyperlipidemia, smoking, and history of myocardial infarction $(p>0.05$ for all). However, there were significant differences among the three groups in the incidences of the multivessel disease, renal insufficiency, Killip grade III/IV, and emergency PCI $(p<0.05$ for all), as well as the levels of high-sensitivity Creactive protein (hs-CRP), cardiac troponin $\mathrm{T}(\mathrm{Tn} T)$, and creatine kinase isoenzyme MB (CK-MB) $(p<0.05$ for all). Comparison of the baseline characteristics of the three groups is shown in Table 1.

3.2. Comparison of In-Hospital Outcomes of the Three Groups. The incidences of severe pump failure, malignant arrhythmia, and death were significantly higher in groups $\mathrm{B}$ and $\mathrm{C}$ than in group $\mathrm{A}(p<0.05)$. The incidences of severe pump failure, malignant arrhythmia, and death were higher in group $\mathrm{C}$ than in group $\mathrm{B}(p<0.05)$. Comparison of the inhospital outcomes of the three groups is shown in Table 2 and Figure 3.

Univariate regression analysis of rough data was performed using severe pump failure, malignant arrhythmia, and TLR as independent variables and death as the dependent variable. The percentage of severe pump failure, malignant arrhythmia, and TLR increased significantly with the increase in BG level in the three groups, thereby leading to increased mortality. Figure 4 shows the mortality trend with the incidence of severe pump failure, showing a linear 
Patients with non ST segment elevation acute coronary syndrome (NSTE-ACS) and hyperglycemia in the department of cardiology of the first affiliated hospital of henan university of science and technology from march 2018 to november $2020(n=754)$

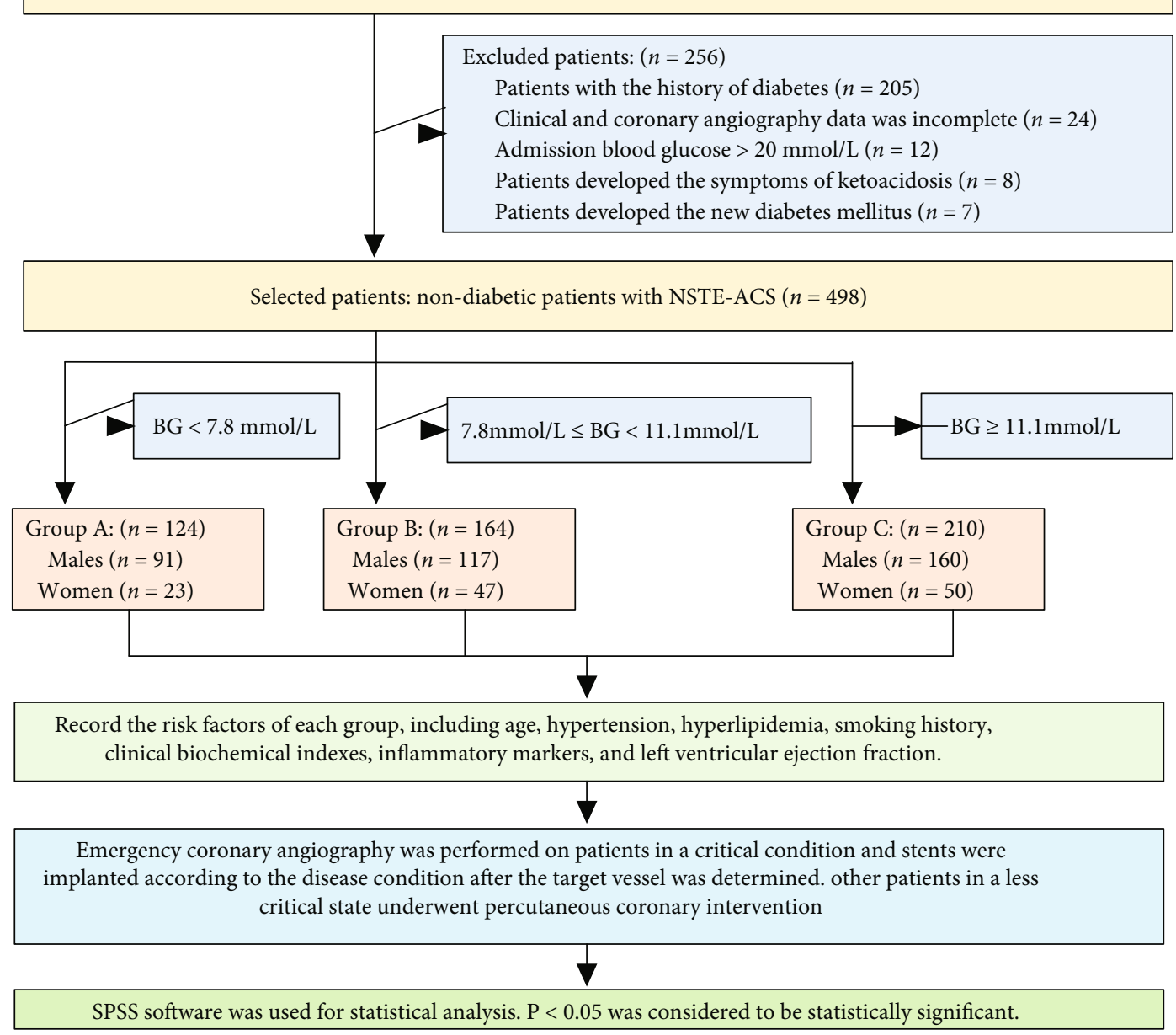

FIGURE 2: Research scheme.

TABLE 1: Comparison of the baseline characteristics of the three groups.

\begin{tabular}{|c|c|c|c|c|c|}
\hline Group $(n)$ & $\mathrm{A}(n=124)$ & $\mathrm{B}(n=164)$ & $\mathrm{C}(n=210)$ & Statistic value & $p$ value \\
\hline Male, $n(\%)$ & $91(74)$ & $117(71)$ & $160(76)$ & 45.217 & 0.81 \\
\hline Age (year) & $65 \pm 8$ & $68 \pm 7$ & $76 \pm 6$ & $19.013^{*}$ & 0.67 \\
\hline Hypertension, $n(\%)$ & $99(80)$ & $141(86)$ & $192(91)$ & 39.571 & 0.85 \\
\hline Hyperlipidemia, $n$ (\%) & $65(52)$ & $81(49)$ & $127(60)$ & 88.263 & 0.37 \\
\hline Smoking, $n(\%)$ & $75(60)$ & $101(62)$ & $153(73)$ & 49.215 & 0.78 \\
\hline Renal inadequacy, $n(\%)$ & $6(4.8)$ & $18(11)$ & $28(13.3)$ & 8.932 & 0.04 \\
\hline Old myocardial infarction, $n(\%)$ & $12(9.7)$ & $28(17.1)$ & $47(22.4)$ & 13.205 & 0.27 \\
\hline Triple vessel disease, $n(\%)$ & $43(34.6)$ & $95(57.9)$ & $181(86.2)$ & 68.213 & $<0.01$ \\
\hline Emergency PCI, $n(\%)$ & $81(65.3)$ & $123(75)$ & $162(77.1)$ & 44.312 & 0.03 \\
\hline Admission blood glucose $(\mathrm{mmol} / \mathrm{L})$ & $6.2 \pm 1.3$ & $9.3 \pm 1.5$ & $17.5 \pm 4.4$ & $23.981^{*}$ & $<0.01$ \\
\hline $\mathrm{TnT}, n(\%)$ & $10(8.1)$ & $63(38.4)$ & $109(51.9)$ & 11.251 & $<0.05$ \\
\hline $\mathrm{CK}-\mathrm{MB}, n(\%)$ & $6(4.8)$ & $40(24.4)$ & $95(45.2)$ & 35.147 & $<0.05$ \\
\hline Killip grading III/IV, $n(\%)$ & $14(11.3)$ & $43(26.2)$ & $103(49.0)$ & 28.102 & 0.02 \\
\hline Hs-CRP (mg/L) & $1.31 \pm 0.93$ & $2.13 \pm 0.25$ & $3.06 \pm 2.61$ & $11.323^{*}$ & $<0.01$ \\
\hline
\end{tabular}

Note: ${ }^{*} F$ value. TnT: troponin T; CK-MB: creatine kinase isoenzyme MB; hs-CRP: high-sensitivity C-reactive protein. 
TABLE 2: Comparison of in-hospital outcomes of the three groups.

\begin{tabular}{lccccc}
\hline Group & Number & Severe pump failure, $n(\%)$ & Malignant arrhythmia, $n(\%)$ & Target lesion revascularization, $n$ (\%) & Death, $n(\%)$ \\
\hline A & 124 & $14(11.3)$ & $10(8.1)$ & $4(3.2)$ & $2(1.6)$ \\
B & 164 & $45(27.4)^{*}$ & $19(11.6)^{*}$ & $8(4.9)^{*}$ & $12(7.3)^{*}$ \\
C & 210 & $79(37.6)^{*}$ & $53(25.2)^{* \Delta}$ & $14(6.5)^{*}$ & $26(12.4)^{* \Delta}$ \\
\hline
\end{tabular}

Note: * comparison with group A and ${ }^{\triangle}$ comparison with group B; $p<0.05$.

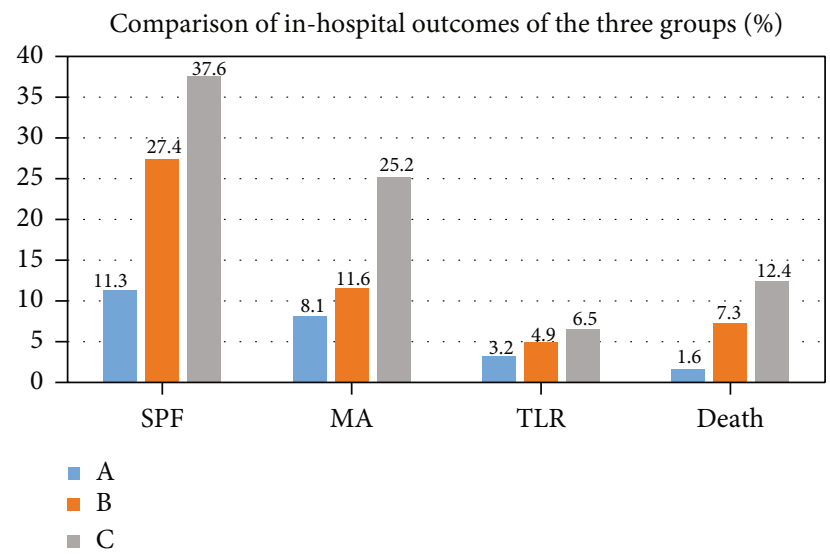

Figure 3: Comparison of the in-hospital outcomes of the three groups.

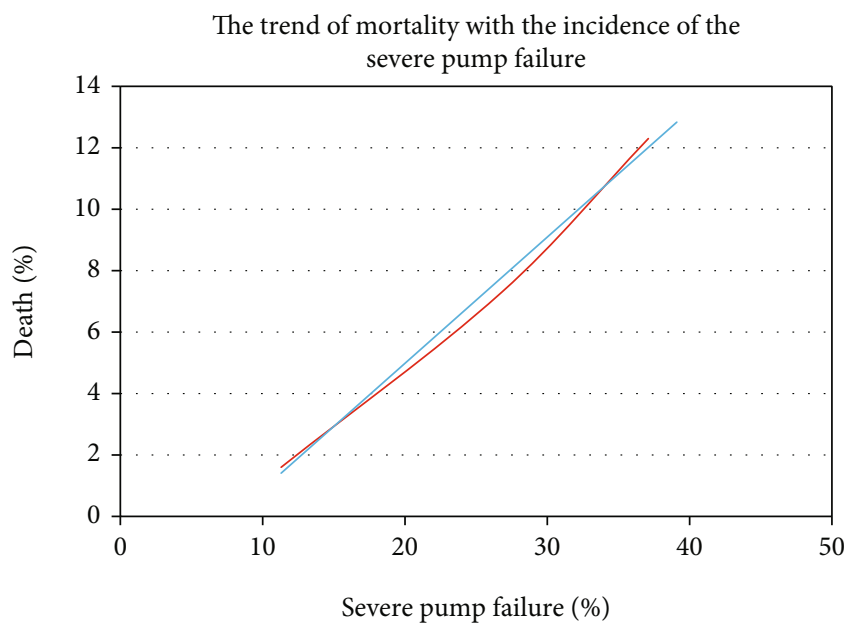

FIgURE 4: Mortality trend with the incidence of severe pump failure.

increase. In Figure 4, red line indicates the univariate regression analysis curve based on the rough data, and blue line indicates the linear prediction trend line for the rough data.

3.3. Risk Factors of In-Hospital Death. Logistic regression analysis was performed with in-hospital death as the dependent variable and the previously identified risk factors as independent variables. The results showed that hyperglycemia, age, renal insufficiency, and severe pump failure were risk factors of in-hospital death. Results of the multivariate logistic regression analysis of in-hospital death are shown in Table 3.

\section{Discussion}

Cardiovascular complications are the main cause of death in diabetic patients $[7,8]$. Previous studies showed that admission hyperglycemia was an independent risk factor for poor prognosis of ACS patients, irrespective of whether or not they had diabetes $[9,10]$. Pasquale first showed that hyperglycemia was associated with adverse outcomes and increased risk of restenosis in ST-elevation myocardial infarction patients without diabetes mellitus [11]. Studies have found that admission hyperglycemia was the greatest risk factor for patients with acute myocardial infarction without diabetes. The 30-day mortality rate of patients without diabetes increased when the admission BG level exceeded $6.1 \mathrm{mmol} / \mathrm{L}$, while the admission BG threshold for the 30-day mortality rate was higher in diabetic patients. Additionally, the increased risk of death associated with high BG level was not limited to known diabetic patients; rather, the mortality rate of patients without diabetes was higher than that of diabetic patients $[12,13]$. Yacov et al. reported that admission hyperglycemia was an independent risk factor for acute kidney injury in nondiabetic ST-segment elevation myocardial infarction patients undergoing primary PCI [14]. Stella et al. performed a meta-analysis on the relationship between admission hyperglycemia and myocardial infarct size using cardiovascular magnetic resonance imaging (CMRI) and found that the size of myocardial infarct detected on cardiovascular magnetic resonance positively correlated with admission hyperglycemia in patients with acute myocardial infarction [15]. Ozge et al. reported that elevated admission BG level attenuated the coronary collateral flow in patients with ST-elevation myocardial infarction [16]. Satoshi et al. pointed out that glycemic variability was associated with myocardial damage after PCI in nondiabetic ST-segment elevation myocardial infarction patients [17].

The findings of our study confirmed that the incidence of severe pump failure, malignant arrhythmia, and death in NSTE-ACS patients without diabetes significantly increased with the increase in BG level at admission. The multivariate analysis showed that admission hyperglycemia was a strong risk factor for adverse outcomes in NSTE-ACS patients. The prognosis of NSTE-ACS patients was related to their BG level after treatment. Although intensive hypoglycemia is not required, BG should be controlled within a reasonable range.

It is necessary to determine the pathophysiological mechanism underlying the poor prognosis of ACS patients with hyperglycemia. First, several physiological studies have confirmed that hyperglycemia causes vascular damage and cardiac myocyte death through different molecular mechanisms [18]. Fabio et al. studied hyperglycemia in ACS 
TABLE 3: Multivariate logistic regression analysis of in-hospital death.

\begin{tabular}{lccc}
\hline Item & Odds ratio & $95 \%$ CI & $p$ value \\
\hline Age & 1.03 & $(0.91,1.14)$ & 0.23 \\
Hyperglycemia at admission & 1.81 & $(1.26,2.41)$ & $<0.01$ \\
Killip grading III/IV & 2.16 & $(1.03,3.96)$ & 0.02 \\
Renal insufficiency & 1.12 & $(1.03,1.21)$ & 0.04 \\
\hline
\end{tabular}

patients and showed that the collateral circulation decreased and infarct size increased in severely hyperglycemic patients [19]. Fang et al. studied in-hospital peak glycemia for predicting no-reflow phenomenon in diabetic patients with ST-elevation myocardial infarction treated with primary PCI and concluded that spontaneous reperfusion rate of hyperglycemia combined with acute ST-segment elevation myocardial infarction was low [20]. Microvascular dysfunction has also been confirmed in acute myocardial infarction patients with hyperglycemia in the study of Simsek et al. that evaluated the association of acute-to-chronic glycemic ratio and no reflow in patients with ST-segment elevation myocardial infarction undergoing primary PCI [21]. Shock index on admission was associated with coronary slow/no reflow in patients with acute myocardial infarction undergoing emergent PCI. Wang et al. found a higher incidence of no blood flow in patients with hyperglycemia after successful reperfusion [22].

Second, hyperglycemia creates a prothrombotic state. In acutely hyperglycemic mice, the level of tissue plasminogen activator was decreased and the level of plasminogen activation inhibitor was increased. Hyperglycemia in type 2 diabetic patients (abnormal glycemic clamp technique) was associated with increased activity of thromboxane $A_{2}$ $\left(\mathrm{TXA}_{2}\right)$ and von Willebrand factor. Acute hyperglycemia caused fibrinogen $t_{1 / 2}$ to decrease and induced platelet aggregation, thereby increasing the levels of fibrinogen A, prothrombin, and factor VII levels. These changes indicate a prothrombotic state. Third, increased BG level was accompanied by increased vascular inflammatory markers $[23,24]$. In vivo and in vitro studies showed that hyperglycemia was associated with increased hs-CRP, cytokine 26 , and tumor necrosis factor $\alpha$ levels. In our study, hyperglycemia significantly correlated with increased inflammatory markers, which increased the incidence of adverse cardiac events through the inflammatory response. In addition, higher BG levels correlated with high levels of free fatty acids, greater insulin resistance, and more serious impairment of myocardial glucose utilization in ACS patients. This increased the oxygen consumption, potentially aggravating myocardial ischemia. At the same time, the increased levels of free fatty acids can induce malignant ventricular arrhythmia, which may be one reason for the increased occurrence of inhospital cardiac arrhythmia with hyperglycemia $[25,26]$.

Therefore, hyperglycemia has different effects on the prognosis of patients with diabetes or undiagnosed diabetes. Hyperglycemia is more predictive of adverse events in patients with undiagnosed diabetes compared to those with diagnosed diabetes. Although the pathophysiological mechanism underlying this phenomenon is unknown, there are several explanations. Some undiagnosed diabetic patients, especially those with severe hyperglycemia, may be at high risk because they have never been treated for diabetes. In addition, in patients with unknown diabetes and hyperglycemia, when acute myocardial infarction occurs, even if blood glucose was significantly elevated, insulin therapy was rarely used. In view of the possible beneficial effect of insulin on myocardial ischemia, this difference in treatment may explain the different prognosis. Finally, it is possible that similar BG level may represent a more serious condition in unknown diabetic patients. There are still many gaps in understanding the relationship between hyperglycemia and the adverse prognosis. Further studies are needed to confirm whether hyperglycemia is an indicator of high mortality.

\section{Research Limitations}

Some limitations of this study should be considered. First, this is a single-center observational study with a small sample size. Second, in our analysis, intracoronary stenosis was determined on angiography, which may be affected by many factors. In the future, more reliable methods should be used to determine the intracoronary stenosis. Third, unmeasured residual confounding factors that may affect the evaluation of coronary stenosis may exist. Finally, the relatively small study population may theoretically lead to selection bias due to the strict inclusion and exclusion criteria. The conclusions drawn from our findings cannot be extrapolated to the patients excluded from the study.

\section{Data Availability}

The data used to support the findings of this study are included within the article.

\section{Conflicts of Interest}

All of the authors declare that they do not have any competing interests.

\section{Acknowledgments}

The authors are grateful for the financial support provided by the National Natural Science Foundation of China (grant no. 51805151), the Key Scientific Research Project of the University of Henan Province of China (grant no. 21B460004), and the Medical Education Research Project of Henan Province of China (grant no. Wjlx2021397).

\section{References}

[1] E. J. Kim, M. H. Jeong, J. H. Kim et al., "Clinical impact of admission hyperglycemia on in-hospital mortality in acute myocardial infarction patients," International Journal of Cardiology, vol. 236, no. 1, pp. 9-15, 2017.

[2] J.-W. Chung, Y.-S. Park, J.-E. Seo et al., "Clinical impact of dysglycemia in patients with an acute myocardial Infarction," 
Diabetes \& Metabolism Journal, vol. 45, no. 2, pp. 270-274, 2021.

[3] Q. Zhao, T. Y. Zhang, Y. J. Cheng et al., "Prognostic significance of relative hyperglycemia after percutaneous coronary intervention in patients with and without recognized diabetes," Current Vascular Pharmacology, vol. 19, no. 1, pp. 91101, 2020.

[4] M. Shahid, H. Zarif, M. S. Farid, M. S. Abid, B. Akhtar, and M. R. Khan, "Prognostic value of hyperglycemia on admission on in-hospital outcomes in patients presenting with STelevation myocardial infarction," Cureus, vol. 12, no. 2, article e7024, 2020.

[5] X. S. Ding, S. S. Wu, H. Chen, X. Q. Zhao, and H. W. Li, "High admission glucose levels predict worse short-term clinical outcome in non-diabetic patients with acute myocardial infraction: a retrospective observational study," BMC Cardiovascular Disorders, vol. 19, no. 1, p. 163, 2019.

[6] C. Y. Cui, M. G. Zhou, L. C. Cheng et al., "Admission hyperglycemia as an independent predictor of long-term prognosis in acute myocardial infarction patients without diabetes: a retrospective study," Journal of Diabetes Investigation, vol. 12, no. 7, pp. 1244-1251, 2021.

[7] N. A. Campos and G. A. Palacios, "Cardiac rehabilitation in patients with diabetes," Panminerva Medica, vol. 63, no. 2, pp. 184-192, 2021.

[8] M. Adil, I. Khan, Z. Hassan, S. A. Habib, M. S. Jibran, and T. Nawaz, "One year outcomes after percutaneous coronary intervention in diabetics with stable ischemic heart disease: a single-center comparative study," Cureus, vol. 13, no. 1, 2021.

[9] G. Koraćević, S. Mićić, M. Stojanović et al., "Single prognostic cut-off value for admission glycemia in acute myocardial infarction has been used although high-risk stems from hyperglycemia as well as from hypoglycemia (a narrative review)," Primary Care Diabetes, vol. 14, no. 6, pp. 594-604, 2020.

[10] T. Y. Chai, M. McLean, V. W. Wong, and N. W. Cheung, "Glycaemic variability is associated with adverse cardiovascular outcomes in patients hospitalised with an acute myocardial infarction," Journal of Clinical and Translational Endocrinology, vol. 18, no. 1, p. 100203, 2019.

[11] P. Mone, J. Gambardella, F. Minicucci, A. Lombardi, C. Mauro, and G. Santulli, "Hyperglycemia drives stent restenosis in STEMI patients," Diabetes Care, vol. 44, no. 11, pp. e192-e193, 2021.

[12] M. Kosiborod, "Hyperglycemia in acute coronary syndromes: from mechanisms to prognostic implications," Endocrinology \& Metabolism Clinics of North America, vol. 47, no. 1, pp. 185-202, 2018.

[13] P. Deedwania, M. Kosiborod, E. Barrett et al., "Hyperglycemia and acute coronary syndrome: a scientific statement from the American Heart Association Diabetes Committee of the Council on Nutrition, Physical Activity, and Metabolism," Anesthesiology, vol. 117, no. 12, pp. 1610-1619, 2008.

[14] Y. Shacham, A. Gal-Oz, E. Leshem-Rubinow et al., "Admission glucose levels and the risk of acute kidney injury in nondiabetic ST segment elevation myocardial infarction patients undergoing primary percutaneous coronary intervention," Cardiorenal Medicine, vol. 5, no. 3, pp. 191-198, 2015.

[15] S. Pak, Y. Yatsynovich, and J.-P. Markovic, “A meta-analysis on the correlation between admission hyperglycemia and myocardial infarct size on CMRI," Hellenic Journal of Cardiology, vol. 59, no. 3, pp. 174-178, 2018.
[16] O. Kurmus, T. Aslan, B. Ekici et al., "Impact of admission blood glucose on coronary collateral flow in patients with ST-elevation myocardial infarction," Cardiology Research and Practice, vol. 2018, Article ID 4059542, 5 pages, 2018.

[17] S. Oka, J. Deyama, K. Umetani et al., "Glycemic variability is associated with myocardial damage in nondiabetic patients with ST-elevation myocardial infarction," Cardiovascular endocrinology \& metabolism, vol. 7, no. 2, pp. 47-53, 2017.

[18] F. C. Sasso, L. Rinaldi, N. Lascar et al., "Role of tight glycemic control during acute coronary syndrome on CV outcome in type 2 diabetes," Journal of Diabetes Research, vol. 2018, Article ID 3106056, 8 pages, 2018.

[19] F. Angeli, G. Reboldi, C. Poltronieri et al., "Hyperglycemia in acute coronary syndromes: from mechanisms to prognostic implications," Therapeutic Advances in Cardiovascular Disease, vol. 9, no. 6, pp. 412-424, 2015.

[20] F. Liu, R. Huang, Y. Li, S. Zhao, Y. Gong, and Z. Xu, "In-hospital peak glycemia in predicting no-reflow phenomenon in diabetic patients with STEMI treated with primary percutaneous coronary intervention," Journal of Diabetes Research, vol. 2021, Article ID 6683937, 7 pages, 2021.

[21] B. Şimşek, T. Çınar, V. Ozan et al., "The association of acuteto-chronic glycemic ratio with no-reflow in patients with STsegment elevation myocardial infarction undergoing primary percutaneous coronary intervention," Kardiologia Polska, vol. 79, no. 2, pp. 170-178, 2021.

[22] Q. Wang, H. Shen, H. Mao, F. Yu, H. Wang, and J. Zheng, "Shock index on admission is associated with coronary slow/ no reflow in patients with acute myocardial infarction undergoing emergent percutaneous coronary intervention," Journal of Interventional Cardiology, vol. 2019, article 7873468, 7 pages, 2019.

[23] T. V. Fiorentino, A. Prioletta, P. Zuo, and F. Folli, "Hyperglycemia-induced oxidative stress and its role in diabetes mellitus related cardiovascular diseases," Current Pharmaceutical Design, vol. 19, no. 32, pp. 5695-5703, 2013.

[24] K. Yahagi, F. D. Kolodgie, C. Lutter et al., "Pathology of human coronary and carotid artery atherosclerosis and vascular calcification in diabetes mellitus," Arteriosclerosis, Thrombosis, and Vascular Biology, vol. 37, no. 2, pp. 191-204, 2017.

[25] M. E. Cerf, "Cardiac glucolipotoxicity and cardiovascular outcomes,” Medicina-Lithuania, vol. 54, no. 5, 2018.

[26] I. Evangelista, R. Nuti, T. Picchioni, F. Dotta, and A. Palazzuoli, "Molecular dysfunction and phenotypic derangement in diabetic cardiomyopathy," International Journal of Molecular Sciences, vol. 20, no. 13, p. 3264, 2019. 